\title{
Magnetosonic waves in a quantum plasma with arbitrary electron degeneracy
}

\author{
Fernando Haas* \\ Physics Institute, Federal University of Rio Grande do Sul, CEP 91501-970, Avenida Bento Gonçalves 9500, \\ Porto Alegre, Rio Grande do Sul, Brazil \\ Shahzad Mahmood \\ Theoretical Physics Division (TPD), PINSTECH, P.O. Nilore Islamabad 44000, Pakistan
}

(Received 16 March 2018; published 22 June 2018)

\begin{abstract}
Using a two-species quantum hydrodynamic model, we derive the quantum counterpart of magnetosonic waves, in a plasma with arbitrary degree of degeneracy and taking into account quantum diffraction effects due to the matter-wave character of the charge carriers. The weakly nonlinear aspects of the associated quantum magnetosonic wave are accessed by means of perturbation theory, with the derivation of a nonlinear evolution equation admitting solitons, namely, the Korteweg-de Vries equation. The degeneracy and quantum diffraction effects on soliton propagation are determined. A qualitative change on weakly nonlinear magnetosonic waves appears when quantum diffraction matches certain conditions, producing shock solutions instead of solitons, within the approximation level. We also include explicit numeric estimates and a discussion on the coupling (nonideality) parameter for quantum plasmas with intermediate degeneracy degree.
\end{abstract}

DOI: 10.1103/PhysRevE.97.063206

\section{INTRODUCTION}

The study of nonlinear magnetohydrodynamic (MHD) waves in plasmas has gained interest due to its wide range of application from astrophysical to laboratory plasmas. The theory of MHD waves in infinite conducting medium was first developed by Alfvén [1] with its application to sunspots, coronal heating, particle acceleration, and generation of cosmic radiation. The Alfvén and magnetosonic waves are the fundamental modes of MHD in plasmas. The Alfvén wave is a transverse wave, which propagates parallel to the magnetic field without fluid density perturbation. The magnetosonic wave is partially longitudinal due to the plasma compression and transverse due to the magnetic field lines compression [2]. The magnetosonic wave is also named the fast MHD wave as its speed is greater than the Alfvén wave speed.

Recently, there has been great interest in studying collective modes in quantum plasmas due to their applications in semiconductors, nanoscale electromechanical structures, laser plasma interactions, and dense astrophysical plasmas; see [3-5] and references therein. The quantum or degeneracy effects become important in plasmas when the de Broglie wavelength associated with electrons/ions becomes of the order of the average interparticle distance. The quantum effects arise by both the Pauli exclusion principle (for fermions like electrons and protons) and the Heisenberg uncertainty principle due to wavelike nature of the particles. The collective motion of quantum particles in magnetic fields can be studied using quantum magnetohydrodynamic (QMHD) theory. The linear and nonlinear MHD waves in fully degenerate plasmas

\footnotetext{
*fernando.haas@ufrgs.br

${ }^{\dagger}$ shahzadm100@gmail.com
}

have been investigated already by a number of authors with their application to interiors of massive planets, white dwarfs, and pulsars. Haas [6] first introduced QMHD models, where nonclassical corrections were included through a quantum diffraction parameter. Further, Marklund and Brodin [7] extended the QMHD model by including spin-1/2 effects of degenerate electrons to study low-frequency waves in magnetized quantum plasmas. Magnetosonic solitons were studied in a quantum magnetoplasma, including the quantum Bohm potential and electron spin-1/2 effects using the Sagdeev potential approach [8]. The authors found that the rarefactive magnetosonic solitons propagating with sub-Alfvénic speed are formed due to balance between nonlinearities and quantum tunneling effects. Also a generalized set of nonlinear electromagnetic quantum hydrodynamic equations has been presented, including certain spin and relativistic effects, in Ref. [9]. Nonlinear magnetosonic waves in dense dissipative magnetized plasmas have also been studied using reductive perturbation techniques, leading to the derivation of a Zabolotskaya-Khoklov equation [10].

The equation of state for a degenerate electrons gas is described by a Fermi-Dirac probability distribution characterized by two independent parameters, i.e., the chemical potential and the thermodynamic temperature [11-13]. The main parameter in a near-to-equilibrium Maxwellian electron gas is the thermodynamic temperature. On the other hand, in a fully degenerate electron gas described by a Fermi-Dirac distribution, the energy spread is uniquely determined by the chemical potential, which is the same as the Fermi energy in this case. In this context, it is interesting to investigate the linear and nonlinear magnetosonic wave propagation in full generality, with an equation of state for arbitrarily degenerate electrons in which these two parameters, i.e., chemical potential and thermal temperature, can be equally relevant, 
and where quantum diffraction effects of electrons are also taken into account. Our model is more relevant to systems which are neither strongly degenerate nor close to classical statistics. In other words, it is more pertinent to systems where the Fermi and thermal temperatures are of the same order of magnitude. This situation happens in inertial confinement plasmas in laboratories with particle densities in the range $10^{30}$ to $10^{32} \mathrm{~m}^{-3}$ and thermodynamic temperatures above $10^{7} \mathrm{~K}$ [14]. Moreover, it has applications such as in laboratory simulations of astrophysical dense plasmas which are better represented in the intermediate Maxwellian and degenerate regimes [15] and in ultrasmall semiconductor devices operating in a mixed dilute-dense plasma regime [16]. In a certain sense, systems with an intermediate statistics have a parallel in certain space plasmas and dusty plasma crystals, where the underlying quasi-equilibrium distribution function can be a Maxwellian or a long tail, kappa distribution according to the circumstances [17].

Using classical kinetic theory, and linearizing the VlasovPoisson system around a Fermi-Dirac equilibrium, Maafa [18] was the first to study ion-acoustic and Langmuir waves in plasmas with arbitrary degeneracy of electrons. Recently, Haas and Mahmood [13,19] investigated the linear and nonlinear ion-acoustic waves unmagnetized (one-dimensional soliton) and magnetized (two-dimensional soliton) in dense plasma with arbitrary degeneracy of electrons. The quantum coupling parameter is defined for an arbitrary degenerate plasma, with limitations for the quantum diffraction parameter in quantum hydrodynamic (QHD) models. Using quantum kinetic theory, the low frequency longitudinal response was obtained in electron-ion plasmas, including quantum recoil for the dilute case [20] and then for the arbitrary degenerate case [21] in a Fermi-Dirac equilibrium. Eliasson and Shukla [22] studied the nonlinear quantum electron fluid evolution equations for the Wigner function in terms of a local Fermi-Dirac equilibrium with an arbitrary thermodynamic temperature. The Langmuir waves were then investigated in the high (Maxwellian) and low (fully degenerate) temperature limits. Dubinov et al. [23] studied the nonlinear ion-acoustic waves with arbitrary degeneracy of electrons and ion by considering them as warm Fermi gases.

In the present paper, we analyze the propagation of linear and weakly nonlinear magnetosonic waves in a plasma with arbitrary degeneracy of electrons and with inclusion of Bohm diffraction effects. For this purpose a two-species QHD model is employed. The set of fluid equations and its linear wave analysis are presented in Sec. II. The normalized set of dynamic equations in described in Sec. III, and the derivation of the Korteweg-de Vries ( $\mathrm{KdV}$ ) equation for magnetosonic waves with arbitrary degeneracy of electrons is presented in Sec. IV. Explicit numeric estimates are presented in Sec. V. The conclusions are shown in Sec. VI. Finally, the Appendix is reserved to show the specific form of certain functions obtained in the weakly nonlinear perturbation theory.

\section{SET OF DYNAMIC EQUATIONS AND LINEAR WAVE ANALYSIS}

We consider an electron-ion magnetized quantum plasma which contains classical (due to their large inertia) and cold ions and inertial degenerate electrons with inclusion of the
Bohm potential effect due to their wave nature, together with Fermi pressure. To have more generality, we do not suppose length scales much longer than the electrons skin depth, so that a two-fluid model is more appropriate than QMHD. To study the low frequency magnetosonic waves, our basic equations are written as follows.

The continuity and momentum equations for the nondegenerate ions are written as

$$
\begin{gathered}
\frac{\partial n_{i}}{\partial t}+\nabla \cdot\left(n_{i} \mathbf{v}_{i}\right)=0 \\
\frac{\partial \mathbf{v}_{i}}{\partial t}+\mathbf{v}_{i} \cdot \nabla \mathbf{v}_{i}=\frac{e}{m_{i}}\left(\mathbf{E}+\mathbf{v}_{i} \times \mathbf{B}\right),
\end{gathered}
$$

where $n_{i}$ and $\mathbf{v}_{i}$ are respectively the ionic number density and velocity field, $\mathbf{E}$ and $\mathbf{B}$ denote the electromagnetic field, $m_{i}$ is the ions mass, and $e$ is the elementary charge.

The continuity and momentum equations for inertial degenerate electrons are given by

$$
\begin{gathered}
\frac{\partial n_{e}}{\partial t}+\nabla \cdot\left(n_{e} \mathbf{v}_{e}\right)=0, \\
\frac{\partial \mathbf{v}_{e}}{\partial t}+\mathbf{v}_{e} \cdot \nabla \mathbf{v}_{e}=-\frac{e}{m_{e}}\left(\mathbf{E}+\mathbf{v}_{e} \times \mathbf{B}\right)-\frac{1}{n_{e} m_{e}} \nabla p \\
+\left(\frac{\alpha}{3}\right) \frac{\hbar^{2}}{2 m_{e}^{2}} \nabla\left(\frac{1}{\sqrt{n_{e}}} \nabla^{2} \sqrt{n_{e}}\right),
\end{gathered}
$$

where $n_{e}$ and $\mathbf{v}_{e}$ are respectively the electronic number density and velocity field, $m_{e}$ is the electron mass, $\hbar$ is the reduced Planck constant, $p$ is the electronic fluid pressure, and $\alpha$ is a factor detailed below, introduced to better fit kinetic theory results.

In order to derive the equation of state for electron pressure, consider a local quasi-equilibrium Fermi-Dirac particle distribution function $f=f(\mathbf{v}, \mathbf{r}, t)$ for electrons [13,19,22], given by

$$
f(\mathbf{v}, \mathbf{r}, t)=\frac{A}{1+e^{\beta(E-\mu)}},
$$

where $\beta=1 /\left(\kappa_{B} T\right), E=m_{e} v^{2} / 2, v=|\mathbf{v}|$, and $\mu$ is the chemical potential regarded as a slowly varying function of position $\mathbf{r}$ and time $t . \kappa_{B}$ is the Boltzmann constant and $T$ is the thermodynamic electron temperature. In addition, the normalization condition at equilibrium $\int f d^{3} v=n_{0}$ gives

$$
-\frac{n_{0}}{\mathrm{Li}_{3 / 2}\left(-e^{\beta \mu_{(0)}}\right)}\left(\frac{\beta m_{e}}{2 \pi}\right)^{3 / 2}=2\left(\frac{m_{e}}{2 \pi \hbar}\right)^{3}=A,
$$

where $\mu_{(0)}$ is the equilibrium chemical potential which is related to the equilibrium number density $n_{0}$. The factor 2 in the middle term of the above equality is due to the electron's spin. Equation (6) contains the polylogarithm function $\mathrm{Li}_{v}(-z)$ [24], which for $v>0$ can be defined as

$$
\operatorname{Li}_{v}(-z)=-\frac{1}{\Gamma(\nu)} \int_{0}^{\infty} \frac{s^{\nu-1}}{1+e^{s} / z} d s, \quad v>0,
$$

where $\Gamma(v)$ is the gamma function. For $v<0$ one applies

$$
\operatorname{Li}_{v}(-z)=\left(z \frac{\partial}{\partial z}\right) \operatorname{Li}_{v+1}(-z)
$$

as many times as necessary, where $v+1>0$. 
The scalar pressure follows from the standard definition for an equilibrium with zero drift velocity,

$$
p=\frac{m_{e}}{3} \int f v^{2} d^{3} v,
$$

which yields

$$
p=\frac{n_{e}}{\beta} \frac{\mathrm{Li}_{5 / 2}\left(-e^{\beta \mu}\right)}{\operatorname{Li}_{3 / 2}\left(-e^{\beta \mu}\right)} .
$$

The last term on right-hand side of Eq. (4) is the Bohm potential term which is responsible for quantum tunneling effects. The constant $\alpha$ in the Bohm potential term is included so that fluid and kinetic theories yield the same dispersion relation in the long-wavelength limit, as described in detail in $[13,19]$ and references therein. It can be also shown that

$$
n_{e}=n_{0} \frac{\mathrm{Li}_{3 / 2}\left(-e^{\beta \mu}\right)}{\mathrm{Li}_{3 / 2}\left(-e^{\beta \mu_{(0)}}\right)}
$$

The numerical coefficient $\alpha$ appearing in the Bohm potential term in Eq. (4) is defined in [13],

$$
\alpha=\frac{\mathrm{Li}_{3 / 2}\left(-e^{\beta \mu_{(0)}}\right) \mathrm{Li}_{-1 / 2}\left(-e^{\beta \mu_{(0)}}\right)}{\left[\mathrm{Li}_{1 / 2}\left(-e^{\beta \mu_{(0)}}\right)\right]^{2}},
$$

an is expressed as a function of the equilibrium fugacity $z=\exp \left(\beta \mu_{(0)}\right)$. In the classical limit $(z \ll 1)$ one has $\alpha \approx 1$, while in the full degenerate limit $(z \gg 1)$ one has $\alpha \approx 1 / 3$.

The necessary Maxwell's equations are

$$
\nabla \times \mathbf{E}=-\frac{\partial \mathbf{B}}{\partial t}
$$

and

$$
\nabla \times \mathbf{B}=\mu_{0} \mathbf{j}+\frac{1}{c^{2}} \frac{\partial \mathbf{E}}{\partial t},
$$

where $\mu_{0}$ is the free space permeability and $c$ the speed of light. The current density is given by

$$
\mathbf{j}=e\left(n_{i} \mathbf{v}_{i}-n_{e} \mathbf{v}_{e}\right)
$$

The equilibrium is defined by $n_{e, i}=n_{0}, \mathbf{v}_{e, i}=0, \mathbf{E}=0$, and $\mathbf{B}=B_{0} \hat{z}$, a uniform magnetic field. In what follows, the displacement current in Eq. (14) will be neglected under the assumption that the wave phase velocity is much smaller than the speed of light.

Without loss of generality, we assume that the magnetosonic wave is propagating in the $x$ direction, i.e., $\nabla=\left(\partial_{x}, 0,0\right)$, while the electric field is lying in the $X Y$ plane, i.e., $\mathbf{E}=E_{x} \hat{\imath}+E_{y} \hat{\jmath}$, and the magnetic field is along the $z$ axis, i.e., $\mathbf{B}=B_{0} \hat{k}+$ $B_{z} \hat{k}$, for first-order quantities $E_{x}, E_{y}$ and $B_{z}$. All perturbations are supposed to be proportional to $\exp (i \mathbf{k} \cdot \mathbf{r}-i \omega t)$, where $\mathbf{k}=k \hat{\imath}$ and $\omega$ are the wave vector and the wave frequency, respectively. With this geometry, the linear dispersion relation for magnetosonic waves in a quantum plasma with arbitrary degeneracy of electrons can be written as

$$
\omega^{2}=\left(c_{s}^{2}+\frac{\alpha}{12} \frac{\hbar^{2}}{m_{i} m_{e}} k^{2}\right) k^{2}+\frac{k^{2} v_{a}^{2}}{1+k^{2} \lambda_{e}^{2}},
$$

where the ion-acoustic speed is defined as

$$
c_{s}=\left(\frac{1}{\beta m_{i}} \frac{\operatorname{Li}_{3 / 2}\left(-e^{\beta \mu_{(0)}}\right)}{\operatorname{Li}_{1 / 2}\left(-e^{\beta \mu_{(0)}}\right)}\right)^{1 / 2},
$$

while the Alfvén speed is $v_{A}=B_{0} / \sqrt{\mu_{0} m_{i} n_{0}}$.

In the case $k^{2} \lambda_{e}^{2} \gg 1$, the dispersion relation described in Eq. (16) becomes

$$
\omega^{2}=\left(c_{s}^{2}+\frac{\alpha}{12} \frac{\hbar^{2}}{m_{i} m_{e}} k^{2}\right) k^{2}+\Omega_{i} \Omega_{e},
$$

where $\Omega_{i}=e B_{0} / m_{i}$ and $\Omega_{e}=e B_{0} / m_{e}$ are the ion and electron gyrofrequencies, respectively.

Now in the absence of Bohm potential force in the model, we have

$$
\omega^{2}=c_{s}^{2} k^{2}+\Omega_{i} \Omega_{e} .
$$

In case of strong magnetic field $\left(c_{s}^{2} k^{2} \ll \Omega_{i} \Omega_{e}\right)$, one has lower hybrid oscillations, i.e., $\omega=\sqrt{\Omega_{i} \Omega_{e}}$.

Wave dispersion effects appear through the electron skin depth $\lambda_{e}=\sqrt{c / \omega_{p e}}$, where $\omega_{p e}=\sqrt{n_{0} e^{2} /\left(m_{e} \varepsilon_{0}\right)}$ is the electron plasma frequency (with $\varepsilon_{0}$ being the free space permittivity). Wave dispersion arises due to the second term in the parentheses of Eq. (16), which is due to the Bohm term. In the long-wavelength limit, one simply has the phase velocity

$$
\frac{\omega}{k}=\sqrt{c_{s}^{2}+v_{A}^{2}}
$$

which is the standard magnetosonic wave for $v_{A} \ll c$, but with a modified ion-sound speed. In the dilute plasma case with a small fugacity $z \ll 1$ and ignoring quantum diffraction, we have

$$
\omega^{2}=c_{s}^{2} k^{2}+\frac{k^{2} v_{A}^{2}}{1+k^{2} \lambda_{e}^{2}},
$$

which is the same as Eq. (16) in Ref. [25], where in this case $c_{s}=\sqrt{\kappa_{B} T / m_{i}}$.

In the fully degenerate case, we have $z \gg 1$ and Eq. (16) can be written as

$$
\omega^{2}=\left(c_{s}^{2}+\frac{\hbar^{2}}{36 m_{i} m_{e}} k^{2}\right) k^{2}+\frac{k^{2} v_{A}^{2}}{1+k^{2} \lambda_{e}^{2}},
$$

where the ion-acoustic speed becomes $c_{s}=\sqrt{2 \varepsilon_{F} /\left(3 m_{i}\right)}$, in terms of the electrons Fermi energy $\varepsilon_{F}=\kappa_{B} T_{F}=$ $\left[\hbar^{2} /\left(2 m_{e}\right)\right]\left(3 \pi^{2} n_{0}\right)^{2 / 3}$, which is the same as the equilibrium chemical potential in the deep degenerate situation, with a Fermi temperature $T_{F}$.

By definition, in a fluid treatment kinetic aspects such as finite Larmor radius effects cannot be addressed. In view of the cold ions assumption, this amounts to considering a long wavelength $\lambda$ such that $\lambda \gg v_{T} / \Omega_{e}$, where $v_{T}=\left\langle v^{2}\right\rangle^{1 / 2}$ is the electron thermal speed. Here,

$$
\left\langle v^{2}\right\rangle=\frac{\int f v^{2} d^{3} v}{n}=\frac{3}{m_{e} \beta} \frac{\operatorname{Li}_{5 / 2}\left(-e^{\beta \mu}\right)}{\operatorname{Li}_{3 / 2}\left(-e^{\beta \mu}\right)},
$$

an expression valid for any degeneracy degree. In equilibrium $\left(\mu=\mu_{0}\right)$, in the fully degenerate case one has $\left\langle v^{2}\right\rangle=(3 / 5) v_{F}^{2}$, while on the opposite limit of a dilute plasma one has $\left\langle v^{2}\right\rangle=$ $3 \kappa_{B} T / m$. 


\section{NORMALIZED SET OF DYNAMIC EQUATIONS}

It is convenient to write the above set of equations (1)-(4) and (11) in dimensionless and component form, as follows. The normalization of the number densities is defined by $\tilde{n}_{e, i}=$ $n_{e, i} / n_{0}$, while the normalized fluid velocities are $\tilde{\mathbf{v}}_{e, i}=\mathbf{v}_{e, i} / c_{s}$. Moreover, we use the following normalizations for space, time, and electric and magnetic fields: $\tilde{x}=\omega_{p i} x / c_{s}, \tilde{t}=\omega_{p i} t$, $\tilde{\mathbf{E}}=e \mathbf{E} /\left(m_{i} c_{s} \omega_{p i}\right)$ and $\tilde{\mathbf{B}}=\mathbf{B} / B_{0}$. The ratio of ion-cyclotron to ion plasma frequencies is defined as $\Omega=\Omega_{i} / \omega_{p i}$, where $\Omega_{i}=e B_{0} / m_{i}$ and $\omega_{p i}=\sqrt{n_{0} e^{2} /\left(m_{i} \varepsilon_{0}\right)}$. The dimensionless quantum diffraction parameter is given $[13,19]$ by

$$
H=\frac{\beta \hbar \omega_{p e}}{\sqrt{3}} \times\left(\frac{\mathrm{Li}_{-1 / 2}\left(-e^{\beta \mu_{(0)}}\right)}{\mathrm{Li}_{3 / 2}\left(-e^{\beta \mu_{(0)}}\right)}\right)^{1 / 2} .
$$

Finally, $\delta=m_{i} / m_{e}$ is the ion-electron mass ratio. With these conventions, the ion continuity equation is given by

$$
\frac{\partial \tilde{n}_{i}}{\partial \tilde{t}}+\frac{\partial}{\partial \tilde{x}}\left(\tilde{n}_{i} \tilde{v}_{i x}\right)=0 .
$$

The $x$ and $y$ components of the ion momentum equation are written as

$$
\begin{aligned}
& \frac{\partial \tilde{v}_{i x}}{\partial \tilde{t}}+\tilde{v}_{i x} \frac{\partial}{\partial \tilde{x}} \tilde{v}_{i x}=\tilde{E}_{x}+\Omega \tilde{v}_{i y} \tilde{B}, \\
& \frac{\partial \tilde{v}_{i y}}{\partial \tilde{t}}+\tilde{v}_{i x} \frac{\partial}{\partial \tilde{x}} \tilde{v}_{i y}=\tilde{E}_{y}-\Omega \tilde{v}_{i x} \tilde{B} .
\end{aligned}
$$

The electron continuity equation is given by

$$
\frac{\partial \tilde{n}_{e}}{\partial \tilde{t}}+\frac{\partial}{\partial \tilde{x}}\left(\tilde{n}_{e} \tilde{v}_{e x}\right)=0 .
$$

The $x$ and $y$ components of the electrons momentum equation are written as

$$
\begin{aligned}
& \frac{\partial \tilde{v}_{e x}}{\partial \tilde{t}}+\tilde{v}_{e x} \frac{\partial}{\partial \tilde{x}} \tilde{v}_{e x}=-\delta \tilde{E}_{x}-\delta \Omega \tilde{v}_{e y} \tilde{B} \\
&-\delta \frac{\operatorname{Li}_{1 / 2}\left(-e^{\beta \mu_{(0)}}\right)}{\operatorname{Li}_{1 / 2}\left(-e^{\beta \mu}\right)} \frac{\partial}{\partial \tilde{x}} \tilde{n}_{e} \\
&+\frac{1}{2} \delta H^{2} \frac{\partial}{\partial \tilde{x}}\left(\frac{1}{\sqrt{\tilde{n}_{e}}} \frac{\partial^{2}}{\partial \tilde{x}^{2}} \sqrt{\tilde{n}_{e}}\right), \\
& \frac{\partial \tilde{v}_{e y}}{\partial \tilde{t}}+\tilde{v}_{e x} \frac{\partial}{\partial \tilde{x}} \tilde{v}_{e y}=-\delta \tilde{E}_{y}+\delta \Omega \tilde{v}_{e x} \tilde{B} \\
& \tilde{n}_{e}=\frac{\operatorname{Li}_{3 / 2}\left(-e^{\beta \mu}\right)}{\operatorname{Li}_{3 / 2}\left(-e^{\beta \mu_{(0)}}\right)}
\end{aligned}
$$

The $z$ component of Faraday's law gives

$$
\frac{\partial \tilde{E}_{y}}{\partial \tilde{x}}=-\Omega \frac{\partial \tilde{B}}{\partial \tilde{t}} .
$$

The $x$ and $y$ components of Ampere's law yield

$$
\begin{gathered}
0=\tilde{n}_{i} \tilde{v}_{i x}-\tilde{n}_{e} \tilde{v}_{e x}, \\
\Omega \frac{\partial \tilde{B}}{\partial \tilde{x}}=\frac{c_{s}^{2}}{c^{2}}\left(\tilde{n}_{e} \tilde{v}_{e y}-\tilde{n}_{i} \tilde{v}_{i y}\right) .
\end{gathered}
$$

For simplicity, the tilde $(\sim)$ sign on the dimensionless quantities is not shown in the remaining calculations.

\section{DERIVATION OF A KdV EQUATION FOR MAGNETOSONIC SOLITONS}

In order to derive the $\mathrm{KdV}$ equation for magnetosonic waves with arbitrary degeneracy of electrons, the well known reductive perturbation method is employed [26]. The stretching of spatial and temporal independent variables is described as follows:

$$
\xi=\epsilon^{1 / 2}\left(x-v_{0} t\right), \quad \tau=\epsilon^{3 / 2} t .
$$

Here $\epsilon$ is a small expansion parameter which characterizes the nonlinearity strength and $v_{0}$ is the phase velocity of the wave, to be determined later on.

We expand the dynamical variables in terms of the smallness parameter $\epsilon$ as follows (where $j=e, i$ ):

$$
\begin{aligned}
n_{j} & =1+\epsilon n_{j 1}+\epsilon^{2} n_{j 2}+\cdots, \\
v_{j x} & =\epsilon v_{j x 1}+\epsilon^{2} v_{j x 2}+\cdots, \\
v_{j y} & =\epsilon^{3 / 2} v_{j y 1}+\epsilon^{5 / 2} v_{j y 2}+\cdots, \\
E_{x} & =\epsilon^{3 / 2} E_{x 1}+\epsilon^{5 / 2} E_{x 2}+\cdots, \\
E_{y} & =\epsilon E_{y 1}+\epsilon^{2} E_{y 2}+\cdots, \\
B & =1+\epsilon B_{z 1}+\epsilon^{2} B_{z 2}+\cdots, \\
\mu & =\mu_{(0)}+\epsilon \mu_{1}+\epsilon^{2} \mu_{2}+\cdots .
\end{aligned}
$$

Now we apply the above perturbation scheme in Eqs. (25) to (34). The lowest order of the $x$ component of the ion continuity and momentum equations give the following set of equations:

$$
\begin{gathered}
-v_{0} \frac{\partial n_{i 1}}{\partial \xi}+\frac{\partial v_{i x 1}}{\partial \xi}=0, \\
-v_{0} \frac{\partial v_{i x 1}}{\partial \xi}=E_{x 1}+\Omega v_{i y 1}, \\
E_{y 1}=\Omega v_{i x 1} .
\end{gathered}
$$

Similarly, the lowest-order $\left(\epsilon^{3 / 2}\right)$ terms from the electron continuity and the $x$ and $y$ components of the electron momentum equations are given as follows:

$$
\begin{gathered}
-v_{0} \frac{\partial n_{e 1}}{\partial \xi}+\frac{\partial v_{e x 1}}{\partial \xi}=0 \\
v_{0} \frac{\partial v_{e x 1}}{\partial \xi}=\delta E_{x 1}+\delta \Omega v_{e y 1}+\delta \frac{\partial n_{e 1}}{\partial \xi}, \\
E_{y 1}=\Omega v_{e x 1} .
\end{gathered}
$$

The lowest-order $(\epsilon)$ terms of Eq. (31) give

$$
\mu_{1}=\frac{1}{\beta} \frac{\mathrm{Li}_{3 / 2}\left(-e^{\beta \mu_{(0)}}\right)}{\mathrm{Li}_{1 / 2}\left(-e^{\beta \mu_{(0)}}\right)} n_{e 1} .
$$

The lowest-order $\left(\epsilon^{3 / 2}\right)$ terms from the $z$ component of the Faraday's law give

$$
\frac{\partial E_{y 1}}{\partial \xi}=v_{0} \Omega \frac{\partial B_{z 1}}{\partial \xi} .
$$

The lowest-order $(\epsilon)$ and $\left(\epsilon^{3 / 2}\right)$ terms from the $x$ and $y$ components of Ampere's law, respectively, yield

$$
\begin{gathered}
v_{i x 1}-v_{e x 1}=0, \\
\frac{c_{s}^{2}}{c^{2}}\left(v_{e y 1}-v_{i y 1}\right)=\Omega \frac{\partial B_{z 1}}{\partial \xi} .
\end{gathered}
$$


Using the plasma approximation, the lowest-order terms of the quasi-neutrality condition give $n_{i 1} \simeq n_{e 1}$.

The normalized phase velocity in the long wavelength approximation, obtained from Eqs. (36) to (45), is given by

$$
v_{0}= \pm \sqrt{\frac{v_{A}^{2}}{c_{s}^{2}}+1}
$$

which is the same as Eq. (20) for a dispersionless wave. Without loss of generality, the positive sign will be adopted.

The higher order set of equations is given as follows:

$$
\begin{gathered}
v_{0} \frac{\partial n_{i 2}}{\partial \xi}-\frac{\partial v_{i x 2}}{\partial \xi}=f_{1}, \\
v_{0} \frac{\partial n_{e 2}}{\partial \xi}-\frac{\partial v_{e x 2}}{\partial \xi}=f_{2}, \\
v_{0} \frac{\partial v_{i x 2}}{\partial \xi}+E_{x 2}+\Omega v_{i y 2}=f_{3}, \\
E_{y 2}-\Omega v_{i x 2}=f_{4}, \\
v_{0} \frac{\partial v_{e x 2}}{\partial \xi}-\delta E_{x 2}-\delta \Omega v_{e y 2}-\delta \frac{\partial n_{e 2}}{\partial \xi}=f_{5}, \\
\delta E_{y 2}-\delta \Omega v_{e x 2}=f_{6}, \\
\frac{\partial E_{y 2}}{\partial \xi}-\Omega v_{0} \frac{\partial B_{z 2}}{\partial \xi}=f_{7}, \\
v_{i x 2}-v_{e x 2}=f_{8}, \\
\frac{c_{s}^{2}}{c^{2}}\left(v_{i y 2}-v_{e y 2}\right)+\Omega \frac{\partial B_{z 2}}{\partial \xi}=f_{9},
\end{gathered}
$$

where the expression of $f_{1}$ to $f_{9}$ are given in the Appendix. Using the above set of equations, we find that

$$
\frac{f_{2}}{v_{0}}+f_{3}+\frac{f_{5}}{\delta}-\frac{\Omega c^{2}}{c_{s}^{2}} f_{9}-\frac{\Omega c^{2}}{v_{0} c_{s}^{2}} f_{7}+\frac{1}{\delta}\left(\frac{\Omega c^{2}}{v_{0} c_{s}^{2}}\right) \frac{\partial f_{6}}{\partial \xi}=0 .
$$

Equation (46) has been used in the derivation of Eq. (56).

In order to express the first-order quantities $v_{i x 1}, n_{i 1}, n_{e 1}$, $B_{z 1}, E_{x 1}, E_{y 1}, v_{i y 1}$, and $v_{e y 1}$ in terms of $v_{e x 1}$, we first solve Eqs. (50) and (52), which gives a relation between electron and ion momentum along the $y$ axis i.e., $v_{e y 1}=-\delta v_{i y 1}$. Using this relation and Eqs. (36)-(45), the variables $v_{i x 1}, n_{i 1}, n_{e 1}$, $B_{z 1}, E_{x 1}, E_{y 1}, v_{i y 1}$, and $v_{e y 1}$ in terms of $v_{e x 1}$ are written as

$$
\begin{gathered}
E_{y 1}=\Omega v_{e x 1}, \\
v_{i x 1}=v_{e x 1}, \\
n_{i 1}=n_{e 1}=\frac{v_{e x 1}}{v_{0}}, \\
B_{z 1}=\frac{v_{e x 1}}{v_{0}}, \\
E_{x 1}=-\frac{1}{v_{0} \delta}\left(v_{0}^{2} \delta+1\right) \frac{\partial v_{e x 1}}{\partial \xi} \approx-v_{0} \frac{\partial v_{e x 1}}{\partial \xi}, \\
v_{i y 1}=\frac{1}{\Omega v_{0} \delta} \frac{\partial v_{e x 1}}{\partial \xi}, \\
v_{e y 1}=\frac{1}{\Omega v_{0}}\left(v_{0}^{2}-1\right) \frac{\partial v_{e x 1}}{\partial \xi} .
\end{gathered}
$$

In Eq. (61), the approximation comes from $\delta \gg 1, v_{0}>1$. Using the above expressions (57)-(63) in Eq. (56), we obtain the $\mathrm{KdV}$ equation in terms of the normalized electron perturbed fluid velocity,

$$
\frac{\partial v_{e x 1}}{\partial \tau}+a v_{e x 1} \frac{\partial v_{e x 1}}{\partial \xi}+b \frac{\partial^{3} v_{e x 1}}{\partial \xi^{3}}=0
$$

where the nonlinearity and dispersion coefficients $a$ and $b$ are defined as

$$
\begin{gathered}
a=\frac{1}{2}\left[3-\frac{1}{v_{0}^{2}}\left(\frac{1}{\delta}+\alpha\right)\right] \approx \frac{1}{2}\left(3-\frac{\alpha}{v_{0}^{2}}\right)>0, \\
b=\frac{1}{2 v_{0}}\left(\frac{\Omega^{2} c^{4}}{\delta c_{s}^{4}}-\frac{H^{2}}{4}\right) .
\end{gathered}
$$

In Eq. (65), the approximation comes from $\delta \gg 1$ and $\alpha$ being of order unity. In addition, the positive sign of $a$ is assured by $\alpha \leqslant 1, v_{0} \geqslant 1$. When the dispersion coefficient $b=0$ at $H=$ $2 \Omega c^{2} /\left(\sqrt{\delta} c_{s}^{2}\right)$, Eq. (64) gives a shock instead of a solitonic solution. Simple algebra yields the shock condition

$$
b=0 \Rightarrow m_{e} m_{i} \lambda_{e}^{2} v_{A}^{2}=\frac{\alpha \hbar^{2}}{12},
$$

within the employed degree of approximation. However, it should be stressed that when the coefficient $b$ goes to zero, the leading dispersion contribution appears at a higher order, including a fifth-order spatial derivative term, yielding the Kawahara equation [27]. This remark is applicable to all shock conditions discussed herein. The corresponding reductive perturbation method would then be necessarily generalized, an issue which will be not considered in the present work.

The limiting cases of Eq. (64) are obtained as follows. In the dilute plasma case $(z \ll 1)$, we have

$$
\begin{aligned}
& \frac{\partial v_{e x 1}}{\partial \tau}+\frac{\left(2 u_{0}^{2}+v_{A}^{2}\right)}{2 u_{0}^{2}} v_{e x 1} \frac{\partial v_{e x 1}}{\partial \xi} \\
& +\frac{\omega_{p i}^{2}}{2 u_{0} c_{s}^{3}}\left(\lambda_{e}^{2} v_{A}^{2}-\frac{\hbar^{2}}{12 m_{e} m_{i}}\right) \frac{\partial^{3} v_{e x 1}}{\partial \xi^{3}}=0
\end{aligned}
$$

where $u_{0}=\sqrt{v_{A}^{2}+c_{s}^{2}}$ is the magnetosonic speed and $c_{s}=\sqrt{\kappa_{B} T / m_{i}}$ is the ion-acoustic speed in this situation. Equation (68) is the same as Eq. (30) of Ref. [28], when written in dimensional form and in terms of the first-order perturbed electron density $n_{e 1}$ using relation (59), and neglecting quantum diffraction.

The shock condition $(b=0)$ in the dilute case can be expressed as

$$
\frac{\Omega_{e}}{\omega_{p e}}=\frac{\hbar \omega_{p e}}{2 \sqrt{3} m_{e} c^{2}}
$$

which depends only on density and magnetic field strength, without a temperature dependence as long as $T \ll T_{F}$, and where $\Omega_{e}=e B_{0} / m_{e}$ is the electron cyclotron frequency. Working out the numbers, Eq. (69) yields

$$
\frac{n_{0}}{B_{0}}=1.49 \times 10^{29} \mathrm{~m}^{-3} \mathrm{~T}^{-1},
$$

in terms of SI units. The equality is quite affordable for typical laboratory and astrophysics environments, and constitutes a 
qualitative structural change due to quantum diffraction effects, within the present level of approximation.

On the other hand, in the dense plasma case $(z \gg 1)$, we have

$$
\begin{aligned}
& \frac{\partial v_{e x 1}}{\partial \tau}+\frac{\left(8 u_{0}^{2}+v_{A}^{2}\right)}{6 u_{0}^{2}} v_{e x 1} \frac{\partial v_{e x 1}}{\partial \xi} \\
& +\frac{\omega_{p i}^{2}}{2 u_{0} c_{s}^{3}}\left(\lambda_{e}^{2} v_{A}^{2}-\frac{\hbar^{2}}{36 m_{e} m_{i}}\right) \frac{\partial^{3} v_{e x 1}}{\partial \xi^{3}}=0,
\end{aligned}
$$

where now $c_{s}=\sqrt{2 \varepsilon_{F} /\left(3 m_{i}\right)}$. The quantum diffraction parameter now is $H=\hbar \omega_{p e} /\left(2 \kappa_{B} T_{F}\right)$. The shock condition is similar to the dilute case:

$$
\frac{\Omega_{e}}{\omega_{p e}}=\frac{\hbar \omega_{p e}}{6 m_{e} c^{2}},
$$

yielding

$$
\frac{n_{0}}{B_{0}}=2.58 \times 10^{29} \mathrm{~m}^{-3} \mathrm{~T}^{-1},
$$

which is also empirically accessible.

The one-soliton solution of Eq. (64) is given by

$$
v_{e x 1}=\Psi_{0} \operatorname{sech}^{2}\left(\frac{\eta}{\Delta}\right) .
$$

where $\eta=\xi-u \tau$, denoting a stationary hump in the comoving frame with a velocity $u$, applying decaying boundary conditions, i.e., $v_{e x 1}, \partial v_{e x 1} / \partial \eta$, and $\partial^{2} v_{e x 1} / \partial \eta^{2}$ going to zero as $\eta \rightarrow \pm \infty$. Here $\Psi_{0}$ is the amplitude and $\Delta$ is the width of magnetosonic soliton:

$$
\Psi_{0}=\frac{3 u}{a}, \quad \Delta=\sqrt{\frac{4 b}{u}} .
$$

It can be seen from Eq. (66) that the amplitude of the soliton depends on quantum degeneracy only, while the width also depends on quantum diffraction.

\section{NUMERICAL ESTIMATES AND COUPLING PARAMETER}

By definition, the present model applies to any degeneracy degree. However, obviously it is more pertinent when the thermal and Fermi temperatures are of the same order. Therefore, in this section we develop the relevant numerical estimates

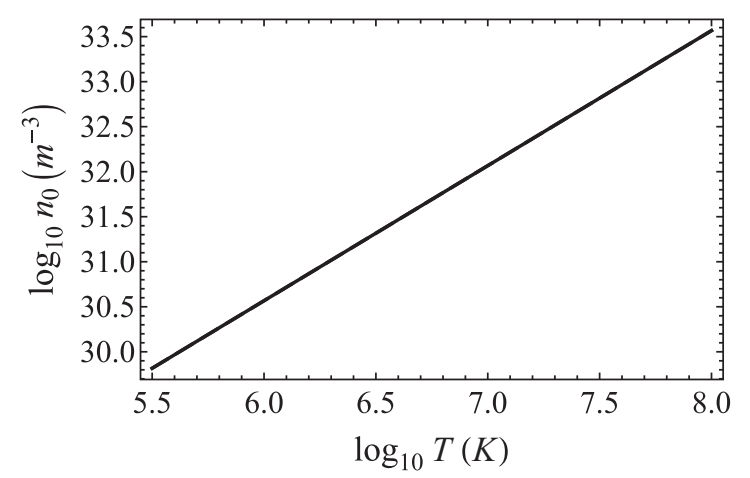

FIG. 1. Plasma number density as a function of temperature, from Eq. (76) and a fixed fugacity $z=1$.

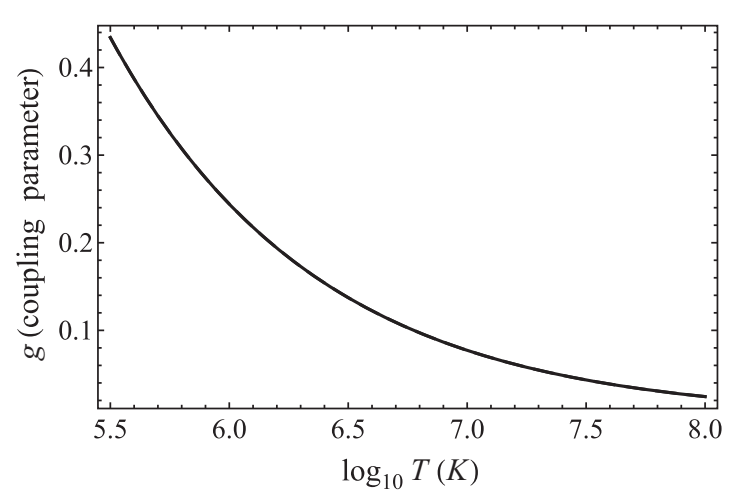

FIG. 2. Plasma coupling parameter $g$ versus electron temperature $T$, from Eq. (77) and fugacity $z=1$.

arising from the previous calculations, setting $T=T_{F}$. In this case, from Eq. (6) it is easy to obtain a fugacity value $z \approx 1$, which we adopt in what follows. Also from Eq. (6), we get

$$
n_{0}=-2\left(\frac{m_{e}}{2 \pi \beta \hbar^{2}}\right)^{3 / 2} \times \operatorname{Li}_{3 / 2}(-z),
$$

which for a fixed fugacity is a function of the temperature only. In this context, we obtain Fig. 1, for typically dense plasmas.

A necessary validity condition for our mean field model disregarding strong interactions between the charge carriers [29] is a small coupling parameter $g$, which is the ratio between the average potential and kinetic energies. The coupling parameter $g$ was determined in Ref. [19] as

$$
g=-\frac{2 \alpha_{F} \sqrt{2 \beta m_{e} c^{2}}}{3(3 \sqrt{\pi})^{1 / 3}} \times \frac{\left[\mathrm{Li}_{3 / 2}^{2}(-z)\right]^{2 / 3}}{\operatorname{Li}_{5 / 2}(-z)},
$$

where $\alpha_{F}=e^{2} /\left(4 \pi \varepsilon_{0} \hbar c\right) \simeq 1 / 137$ is the fine structure constant [30]. We then get Fig. 2, which shows $g<1$ for sufficiently high temperatures.

The increase of the ion-acoustic speed with the electronic temperature (presently with the same numerical value as the Fermi temperature) is shown in Fig. 3. In Fig. 4, we show the decrease in the Alfvén speed due to the increase of the number density and hence the increase of temperature (for $z=1$ ). Typical Alfvén speeds are shown in Fig. 4, for a few magnetic field strengths.

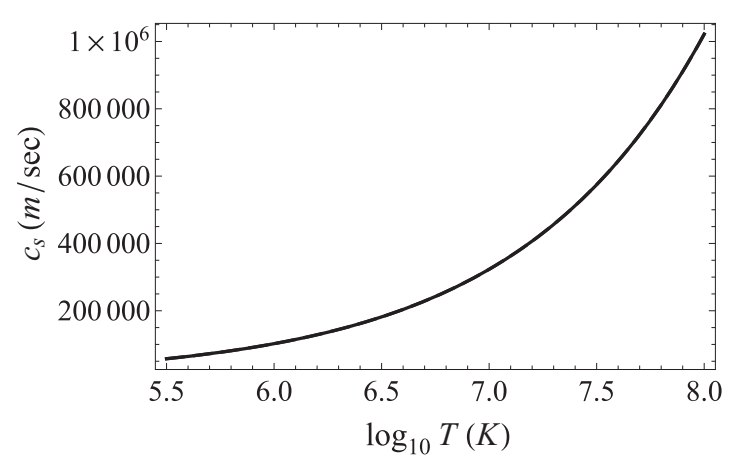

FIG. 3. Ion-acoustic speed $c_{s}$ as a function of temperature, from Eq. (17) and $z=1$. 


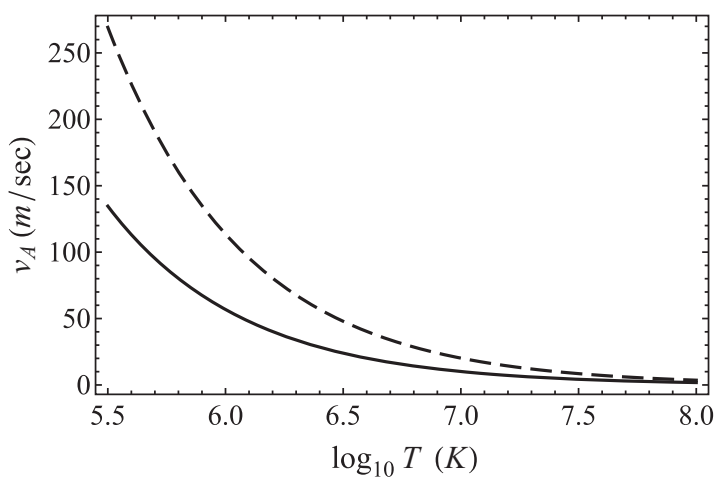

FIG. 4. Alfvén wave speed $v_{A}$ as a function of temperature, for $B_{0}=5 \mathrm{~T}$. (solid curve) and $B_{0}=10 \mathrm{~T}$ (dashed curve), at $z=1$.

The dependence of the quantum diffraction parameter $H$ on the electrons thermal temperature is shown in Fig. 5. It is seen that $H$ attains reasonably large values at least for sufficiently cold plasma.

The variation of the dispersive coefficient $b$ of the $\mathrm{KdV}$ equation (64) for magnetosonic waves at different magnetic field intensities is shown in Fig. 6. It can be seen from the figure that $b<0$ for electron temperatures $T>10^{6} \mathrm{~K}$. In this case only rarefactive magnetosonic solitons structures are formed with $u<0$ since the nonlinearity coefficient $a$ remains positive. The velocity of the nonlinear structure $u$ has to be negative for the dispersive coefficient $b<0$ case to give the real value of the width of the soliton defined in Eq. (75), which means that the speed of the nonlinear rarefactive soliton will be less than the phase speed of the magnetosonic waves, as discussed in detail in Ref. [13]. However, for electron temperature values less than $10^{6} \mathrm{~K}$, the magnetosonic soliton compressive structure is formed as the dispersive coefficient $b>0$ for which $u>0$, and it moves with a speed greater than the speed of the magnetosonic waves in the plasma with arbitrary degeneracy of electrons. It can also be seen from Fig. 6 that with the increase of the magnetic field intensity the range of electron thermal temperature for the formation of magnetosonic dip structures in the higher temperature region is decreased.

Finally, the variations of dimensionless parameter $\Omega=$ $\Omega_{i} / \omega_{p i}$ (ratio of ion-cyclotron to ion plasma frequencies) is

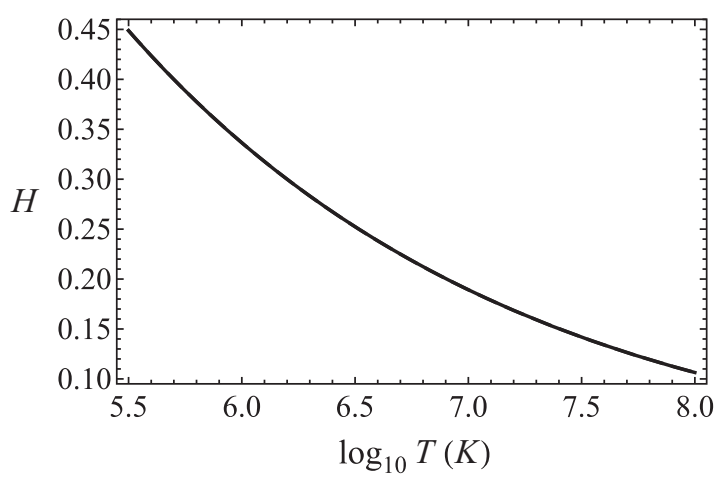

FIG. 5. Quantum diffraction parameter $H$ as a function of temperature, from Eq. (24) and $z=1$.

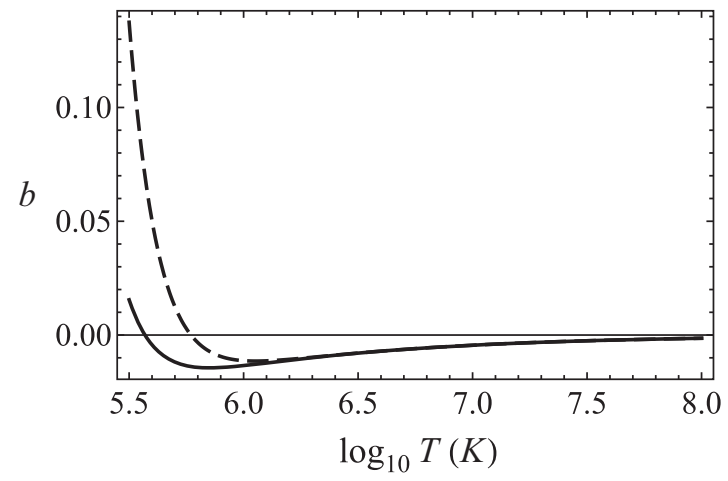

FIG. 6. Nonlinear dispersive coefficient $b$ as a function of temperature, from Eq. (66), for $B_{0}=5 \mathrm{~T}$ (solid curve) and $B_{0}=10 \mathrm{~T}$ (dashed curve), at $z=1$.

plotted in Fig. 7. It can be seen that the value of $\Omega$ decreases with the increase in the electron thermal temperature. However, it obviously increases with the magnetic field intensity, as shown in the same figure.

\section{CONCLUSIONS}

Besides Alfvén waves, magnetosonic waves are the most basic low-frequency excitations propagating in electron-ion magnetized plasmas. We have analyzed the linear and weakly nonlinear propagation of magnetosonic waves in a quantum plasma with arbitrary degeneracy of electrons and with inclusion of matter-wave diffraction effects. Using perturbation theory, we have derived the appropriate nonlinear evolution equation for the propagation of quantum magnetosonic waves. In this case, it is a $\mathrm{KdV}$ equation whose coefficients strongly depend on the degeneracy degree and strength of the quantum diffraction effects. The impact of the quantum degeneracy and diffraction effects on soliton propagation is discussed with explicit numeric estimates for systems in the intermediate range of Maxwell-Boltzmann and Fermi-Dirac statistics. Conditions for shock development, within the present weakly nonlinear limits, were worked out in detail. The validity of the weak coupling assumption was analyzed in concrete cases. Generically, our main results are more relevant for plasmas where the Fermi and thermodynamic temperature are of the

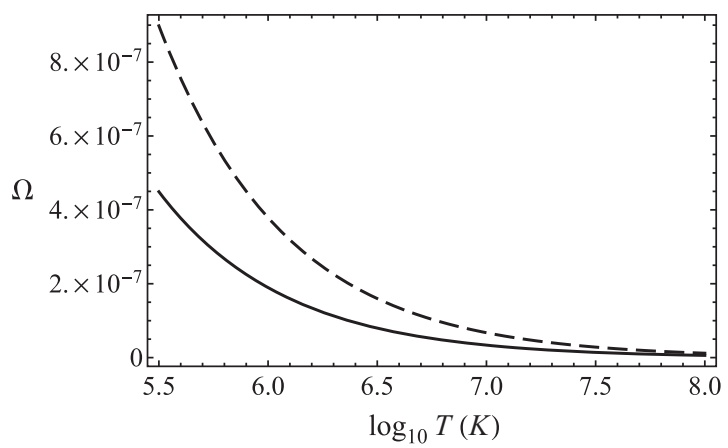

FIG. 7. The ratio $\Omega$ of ion-cyclotron to ion plasma frequencies as a function of temperature, for $B_{0}=5 \mathrm{~T}$ (solid curve) and $B_{0}=10 \mathrm{~T}$ (dashed curve), at $z=1$. 
same order. Finally, we observe that a rigorous treatment of exchange-correlation effects in quantum plasma fluid models at arbitrary degeneracy degree is still unavailable, and is under analysis for future works.

\section{ACKNOWLEDGMENT}

F.H. acknowledges CNPq (Conselho Nacional de Desenvolvimento Científico e Tecnológico) for financial support.

\section{APPENDIX: FUNCTIONS DEFINED AFTER COLLECTING FIRST- AND SECOND-ORDER TERMS FROM PERTURBATION THEORY}

The functions $f_{1}$ to $f_{9}$ defined after Eq. (46) are given as follows:

$$
\begin{aligned}
& f_{1}=\frac{\partial n_{i 1}}{\partial \tau}+\frac{\partial}{\partial \xi}\left(n_{i 1} v_{i x 1}\right), \\
& f_{2}=\frac{\partial n_{e 1}}{\partial \tau}+\frac{\partial}{\partial \xi}\left(n_{e 1} v_{e x 1}\right),
\end{aligned}
$$

$$
\begin{aligned}
f_{3}= & \frac{\partial v_{i x 1}}{\partial \tau}+v_{i x 1} \frac{\partial}{\partial \xi} v_{i x 1}-\Omega v_{i y 1} B_{z 1}, \\
f_{4}= & -v_{0} \frac{\partial v_{i y 1}}{\partial \xi}+\Omega v_{i x 1} B_{z 1}, \\
f_{5}= & \frac{\partial v_{e x 1}}{\partial \tau}+v_{e x 1} \frac{\partial}{\partial \xi} v_{e x 1}+\delta \Omega v_{e y 1} B_{z 1} \\
& -\delta \alpha n_{e 1} \frac{\partial n_{e 1}}{\partial \xi}-\delta \frac{H^{2}}{4} \frac{\partial^{3} n_{e 1}}{\partial \xi^{3}}, \\
f_{6}= & v_{0} \frac{\partial v_{e y 1}}{\partial \xi}+\delta \Omega v_{e x 1} B_{z 1}, \\
f_{7}= & -\Omega \frac{\partial B_{z 1}}{\partial \tau}, \\
f_{8}= & \left(n_{e 1}-n_{i 1}\right) v_{e x 1}, \\
f_{9}= & \frac{c_{s}^{2}}{c^{2}}\left(n_{e 1} v_{e y 1}-n_{i 1} v_{i y 1}\right) .
\end{aligned}
$$

[1] H. Alfvén, Nature (London) 150, 405 (1942).

[2] N. Herlofson, Nature (London) 165, 1020 (1950).

[3] F. Haas, Quantum Plasmas: An Hydrodynamic Approach (Springer, New York, 2011).

[4] S. H. Glenzer, O. L. Landen, P. Neumayer, R. W. Lee, K. Widmann, S. W. Pollaine, R. J. Wallace, G. Gregori, A. Höll, T. Bornath, R. Thiele, V. Schwarz, W.-D. Kraeft, and R. Redmer, Phys. Rev. Lett. 98, 065002 (2007).

[5] A. K. Harding and D. Lai, Rep. Prog. Phys. 69, 2631 (2006).

[6] F. Haas, Phys. Plasmas 12, 062117 (2005).

[7] M. Marklund and G. Brodin, Phys. Rev. Lett. 98, 025001 (2007).

[8] M. Marklund, B. Eliasson, and P. K. Shukla, Phys. Rev. E 76, 067401 (2007).

[9] W. Masood, B. Eliasson, and P. K. Shukla, Phys. Rev. E 81, 066401 (2010).

[10] W. Masood, R. Jhangir, B. Eliasson, and M. Siddiq, Phys. Plasmas 21, 102311 (2014).

[11] R. K. Pathria and P. D. Beale, Statistical Mehanics, 3rd ed. (Elsevier, New York, 2011).

[12] A. E. Dubinov and A. A. Dubinova, Plasma Phys. Rep. 34, 403 (2008).

[13] F. Haas and S. Mahmood, Phys. Rev. E 92, 053112 (2015).

[14] G. Manfredi and J. Hurst, Plasma Phys. Controlled Fusion 57, 054004 (2015).
[15] J. E. Cross, B. Reville, and G. Gregori, Astrophys. J. 795, 59 (2014).

[16] A. Jüngel, Transport Equations for Semiconductors (Springer, Berlin, 2009).

[17] M. A. Hellberg and R. L. Mace, Phys. Plasmas 9, 1495 (2002).

[18] N. Maafa, Phys. Scr. 48, 351 (1993).

[19] F. Haas and S. Mahmood, Phys. Rev. E 94, 033212 (2016).

[20] A. Mushtaq and D. B. Melrose, Phys. Plasmas 16, 102110 (2009).

[21] D. B. Melrose and A. Mushtaq, Phys. Rev. E 82, 056402 (2010).

[22] B. Eliasson and P. K. Shukla, Phys. Scr. 78, 025503 (2008).

[23] A. E. Dubinov, A. A. Dubinova, and M. A. Sazokin, J. Commun. Technol. Electron. 55, 907 (2010).

[24] L. Lewin, Polylogarithms and Associated Functions (North-Holland, New York, 1981).

[25] H. Ur-Rehman, S. Mahmood, and S. Hussain, Phys. Plasmas 24, 012106 (2017)

[26] S. Hussain and S. Mahmood, Phys. Plasmas 24, 032122 (2017).

[27] T. Kawahara, J. Phys. Soc. Jpn. 33, 260 (1972).

[28] Y. Ohsawa and J. Sakai, Astrophys. J. 313, 440 (1987).

[29] S. Ichimaru, Statistical Plasma Physics, Volume II: Condensed Plasmas (Westview, Oxford, 2004).

[30] The rest mass energy $m_{e} c^{2}$ and $\alpha$ were used just to express $g$ in a not so awkward form, although the theory is clearly nonrelativistic. 